\title{
Endoscopic Ultrasonography for Vascular Invasion in Pancreatic Cancer
}

\author{
Seong-Hun Kim ${ }^{1,2,3}$ \\ ${ }^{1}$ Department of Internal Medicine, Chonbuk National University Hospital, Chonbuk National University Medical School, Jeonju, ${ }^{2}$ Research \\ Institute of Clinical Medicine, Chonbuk National University, Jeonju, ${ }^{3}$ Biomedical Research Institute, Chonbuk National University Hospital, \\ Jeonju, Korea
}

See "Diagnostic Ability of Convex-Arrayed Endoscopic Ultrasonography for Major Vascular Invasion in Pancreatic Cancer" by Yuki Fujii, Kazuyuki Matsumoto, Hironari Kato, et al., on page 479-485.

The prognosis in patients with pancreatic cancer is poor with a five-year survival rate of $8 \%-9 \%{ }^{1,2}$ Curative resection is indispensable procedure for achieving a higher survival rate. ${ }^{1,2}$ Surgical resectability of pancreatic cancer can be determined by evaluating vascular invasion, especially in the major arteries (superior mesenteric, celiac, and common hepatic arteries) and the veins (superior mesenteric and portal veins). ${ }^{3,4}$ Although contrast-enhanced computed tomography (CECT) is the standard method for the diagnosis of pancreatic cancer, there are reports that endoscopic ultrasonography (EUS) has some advantages for detecting vascular invasion. ${ }^{5,6}$

In 2006, a systematic review comparing EUS and CECT for pancreatic cancer demonstrated that EUS is the superior technique for tumor detection, tumor staging, and nodal staging, but it has not been concluded which of these is superior in evaluating resectability. ${ }^{7}$ On the other hand, a meta-analysis showed that the sensitivity to diagnosing vascular invasion was higher in EUS (86\%) than in CECT (58\%), ${ }^{5}$ which was

Received: July 20, 2019 Revised: July 30, 2019

Accepted: July 30, 2019

Correspondence: Seong-Hun Kim

Department of Internal Medicine, Chonbuk National University Hospital, Chonbuk National University Medical School, 20 Geonji-ro, Deokjin-gu, Jeonju 54907, Korea

Tel: +82-63-250-2295, Fax: 82-63-254-1609, E-mail: shkimgi@jbnu.ac.kr ORCID: https://orcid.org/0000-0002-7592-8060

(c) This is an Open Access article distributed under the terms of the Creative Commons Attribution Non-Commercial License (http://creativecommons.org/ licenses/by-nc/3.0) which permits unrestricted non-commercial use, distribution, and reproduction in any medium, provided the original work is properly cited. confirmed by a systematic review (EUS 72\% and CT 63\%). In a meta-analysis study, however, there was no significant difference between the sensitivity (CECT $71 \%$ vs. magnetic resonance imaging [MRI] 67\%) and the specificity (CECT $92 \%$ vs. MRI 94\%) for vascular invasion. ${ }^{3}$ Although CECT has better inter-observer agreement, accuracy, and skill, it is now believed that EUS is superior to CECT when evaluating vascular invasion and resectability in pancreatic cancer.

In the current issue, Fujii et al. studied the diagnostic ability of EUS for major vascular invasion in pancreatic cancer, and evaluated the relationship between EUS findings and pathological distance. ${ }^{8}$ The sensitivity, specificity, and accuracy of EUS diagnosis for vascular invasion were $89 \%$, 92\%, and $91 \%$ in the veins and $83 \%, 94 \%$, and $93 \%$ in the arteries, respectively. ${ }^{8}$ The values showed narrow improvement from previous studies, despite the development of EUS. In accordance with the relationship between tumors and major vessels, Fujii et al. classified the EUS findings into four types: type 1, clear invasion, encasement of a vessel by a tumor; type 2 , a tumor that makes contact with a vessel, with loss of the hyperechoic vessel layer; type 3, a tumor that makes contact with a vessel, without loss of the hyperechoic vessel layer; type 4, clear non-invasion, with space between a tumor and a vessel. ${ }^{8}$ The vascular invasion defined by Fujii et al. is far simpler than in previous studies. ${ }^{8}$ According to Buchs et al., the findings of vascular invasion in EUS are as follows: (1) loss of the hyperechoic vessel wall/tumor interface; (2) direct visualization of tumor within the vessel lumen; (3) vascular encasement or 
occlusion; (4) nonvisualization of a major vessel in the presence of collaterals; (5) proximity of the tumor ( $<3 \mathrm{~mm})$ to the vessel; (6) irregularity of the vascular wall. ${ }^{9}$ Between these two definitions, the one by Fujii et al. has the advantage of allowing the clinician to assess the vascular invasion more easily, but has the potential disadvantage of decreased accuracy. ${ }^{8}$ For example, Fujii et al. explained that no vessel invasion is expected when the distance between the main vessel and tumor is $1 \mathrm{~mm}$ or greater by EUS, therefore, there may be more cases that meet the criteria for surgical tumor resection than already discovered. ${ }^{8}$ However, this is a retrospective study involving only a few cases, thereby requiring careful interpretation. For instance, it may have been difficult to clearly distinguish types 2 and 3 from images stored in this study.

In the study by Fujii et al., 5 of 8 cases (positive predictive value $[\mathrm{PPV}]=63 \%$ ) were determined to have major artery invasion in pancreatic cancer by EUS. ${ }^{8}$ In addition, all 3 cases with type 2 were incorrectly diagnosed in the major arteries, ${ }^{8}$ which indicates that the EUS findings of major artery invasion may prevent patients from undergoing surgical resections of resectable tumors. This is similar to previous studies that have shown EUS to be a sensitive diagnostic tool to detect portal and splenic vein invasion in pancreatic cancer; however, regarding artery invasion, a wide range of sensitivity (50\%-100\%), specificity (58\%-100\%), PPV (28\%-100\%), and negative predictive value (18\%-93\%) of EUS has been reported. ${ }^{9,10}$ As mentioned above, CECT is more sensitive than EUS for the detection of arterial invasion.

The authors have noted several limitations of their study including the study design (retrospective, a case-control study), lack of evaluation of the common hepatic artery, having knowledge of the results of different imaging methods before, and the limited amount of tissue available to study. ${ }^{8}$ Despite these limitations, this study is clinically significant as it measures the distance between the tumor and blood vessels in the tissue by EUS.

Recently, EUS-related technologies and equipment have been steadily improving due to the development of contrast-enhanced EUS, EUS elastography, EUS-guided fine needle aspiration, monitor/processing units, and endoscopic technique. ${ }^{1}$ Such development of EUS-related technologies is expected to play an important role in staging by evaluating the vascular invasion in pancreatic cancer.

In conclusion, EUS is a useful tool for evaluating vascular invasion in pancreatic cancer compared to other diagnostic tools. The study by Fujii et al. demonstrated that the accuracy of EUS is excellent for evaluating vascular invasion when the distance between the pancreatic tumor and major blood vessel is $\geq 1 \mathrm{~mm}^{8}$ In addition, the surgical resectability should not be decided by using EUS alone, since EUS has lower PPV than CECT in determining major artery invasion. ${ }^{8}$ According to previous research and the present study, major vein invasion is more accessible by EUS than by CECT, and the ability of evaluation of major artery invasion by EUS is lower than that by CECT. ${ }^{8-10}$ Lastly, further prospective studies including more cases are recommended to confirm the validity of EUS in staging the disease, since major vascular involvement is an important criterion to decide the prognosis and the surgical resectability of pancreatic cancer.

\section{Conflicts of Interest}

The author has no financial conflicts of interest.

\section{REFERENCES}

1. Kitano M, Yoshida T, Itonaga M, Tamura T, Hatamaru K, Yamashita Y. Impact of endoscopic ultrasonography on diagnosis of pancreatic cancer. J Gastroenterol 2019;54:19-32.

2. Wagner M, Redaelli C, Lietz M, Seiler CA, Friess H, Büchler MW. Curative resection is the single most important factor determining outcome in patients with pancreatic adenocarcinoma. Br J Surg 2004;91:586-594.

3. Zhang Y, Huang J, Chen M, Jiao LR. Preoperative vascular evaluation with computed tomography and magnetic resonance imaging for pancreatic cancer: a meta-analysis. Pancreatology 2012;12:227-233.

4. DiMagno EP, Reber HA, Tempero MA. AGA technical review on the epidemiology, diagnosis, and treatment of pancreatic ductal adenocarcinoma. American Gastroenterological Association. Gastroenterology 1999;117:1464-1484.

5. Nawaz H, Fan CY, Kloke J, et al. Performance characteristics of endoscopic ultrasound in the staging of pancreatic cancer: a meta-analysis. JOP 2013;14:484-497.

6. Yang R, Lu M, Qian X, et al. Diagnostic accuracy of EUS and CT of vascular invasion in pancreatic cancer: a systematic review. J Cancer Res Clin Oncol 2014;140:2077-2086.

7. Dewitt J, Devereaux BM, Lehman GA, Sherman S, Imperiale TF. Comparison of endoscopic ultrasound and computed tomography for the preoperative evaluation of pancreatic cancer: a systematic review. Clin Gastroenterol Hepatol 2006;4:717-725; quiz 664.

8. Fujii Y, Matsumoto K, Kato H, et al. Diagnostic ability of convex-arrayed endoscopic ultrasonography for major vascular invasion in pancreatic cancer. Clin Endosc 2019;52:479-485.

9. Buchs NC, Chilcott M, Poletti PA, Buhler LH, Morel P. Vascular invasion in pancreatic cancer: imaging modalities, preoperative diagnosis and surgical management. World J Gastroenterol 2010;16:818-831.

10. Brugge WR, Lee MJ, Kelsey PB, Schapiro RH, Warshaw AL. The use of EUS to diagnose malignant portal venous system invasion by pancreatic cancer. Gastrointest Endosc 1996;43:561-567. 